\title{
Relative effects of open biomass burning and open crop straw burning on haze formation over central and eastern China: modeling study driven by constrained emissions
}

Khalid Mehmood et al.

Correspondence to: Shaocai Yu (shaocaiyu@zju.edu.cn) and Pengfei Li (lpf_zju@163.com)

The copyright of individual parts of the supplement might differ from the CC BY 4.0 License. 

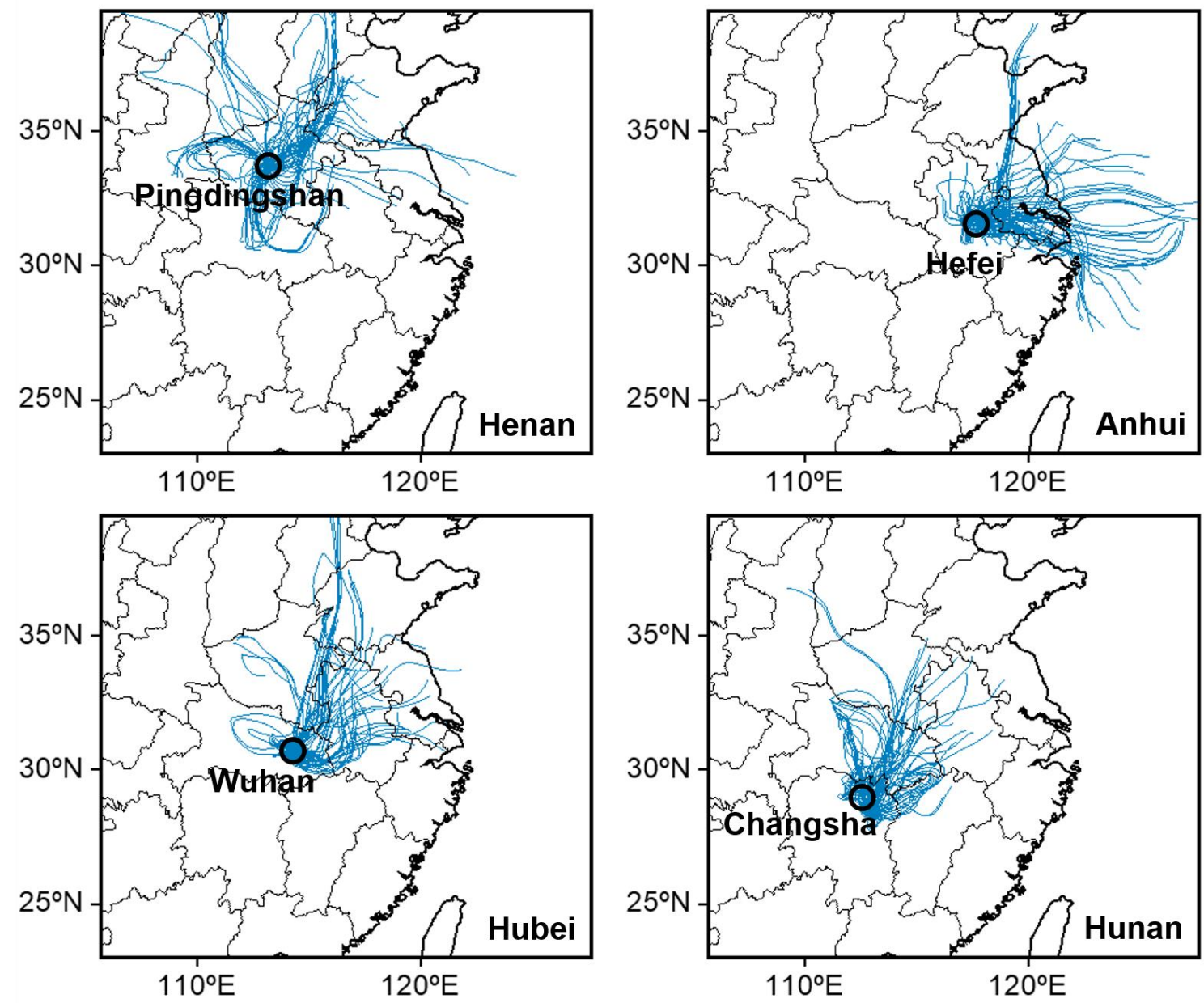

Figure S1. 48h backward trajectories during EP2 for four representative cities (Pingdingshan, Hefei, Wuhan and Changsha) for Henan, Anhui, Hubei and Hunan. 
15 Table $\mathbf{S 1}$. The fire-related parameters (i.e., $\mathbf{B}_{\text {size }}, \mathbf{P}_{\text {topmax }}$, and $\mathbf{P}_{\text {bottommax }}$ ) as a function of the fire size classes.

\begin{tabular}{crrrrr}
\hline Fire Class & 1 & 2 & 3 & 4 & 5 \\
\hline Size & $0 \sim 10$ & $10 \sim 100$ & $100 \sim 1000$ & $1000 \sim 5000$ & $>5000$ \\
$\mathrm{~B}_{\text {size }}$ & 0.4 & 0.6 & 0.75 & 0.85 & 0.9 \\
$\mathrm{P}_{\text {topmax }}$ & 160 & 2400 & 6400 & 7200 & 8000 \\
$\mathrm{P}_{\text {bottommax }}$ & 0 & 900 & 2200 & 3000 & 300 \\
\hline
\end{tabular}


45 Table S2. The activity related parameter (i.e., $B_{\text {hour }}$ ) as a function of hour of day.

\begin{tabular}{crrr}
\hline Hour & \multicolumn{1}{c}{$\mathrm{B}_{\text {hour }}$} & Hour & \multicolumn{1}{c}{$\mathrm{B}_{\text {hour }}$} \\
\hline 0 & 0.03 & 12 & 0.7 \\
1 & 0.03 & 13 & 0.8 \\
2 & 0.03 & 14 & 0.9 \\
3 & 0.03 & 15 & 0.95 \\
4 & 0.03 & 16 & 0.99 \\
5 & 0.03 & 17 & 0.8 \\
6 & 0.03 & 18 & 0.7 \\
7 & 0.03 & 19 & 0.4 \\
8 & 0.06 & 20 & 0.06 \\
9 & 0.1 & 21 & 0.03 \\
10 & 0.2 & 22 & 0.03 \\
11 & 0.4 & 23 & 0.03 \\
\hline
\end{tabular}


Table S3. Detailed information for 5 monitoring sites of $\mathrm{PM}_{2.5}$ composition.

\begin{tabular}{ccccccc}
\hline Station & Province & Latitude $(\mathrm{E})$ & Longitude $(\mathrm{N})$ & Altitude $(\mathrm{m})$ & Station Type & $\mathrm{PM}_{2.5}$ Compositions \\
\hline Changsha & Hunan & 113.06 & 28.21 & 45 & Urban & \\
Qianyanzhou & Jiangxi & 115.07 & 26.75 & 76 & Rural/suburban & \\
Wuxi & Jiangsu & 120.35 & 31.5 & 5 & Urban & $\mathrm{K}^{+}, \mathrm{SO}_{4}{ }^{2-}, \mathrm{NO}_{3}{ }^{-}$, \\
Yucheng & Shandong & 116.6 & 36.95 & 22 & Rural/suburban & \\
Hefei & Anhui & 117.27 & 31.86 & 24 & Und $\mathrm{NH}_{4}{ }^{-}$ & \\
\hline
\end{tabular}


Table S4. Original OBB and OCSB emissions estimated in FINNv1.5 for EP1 EP3 over CEC (Units: million moles for NMVOCs, SO 2 , CO, NH3, and NOx and tons for EC, OC, primary $\mathrm{PM}_{2.5}$ and $\left.\mathrm{PM}_{10}\right)$.

\begin{tabular}{|c|c|c|c|c|c|c|c|c|c|c|c|c|c|c|c|c|c|c|c|}
\hline \multirow{2}{*}{ Episode } & \multirow{2}{*}{ Provinces- } & \multicolumn{9}{|c|}{ OBB } & \multicolumn{9}{|c|}{ OCSB } \\
\hline & & NMVOCs & $\mathrm{SO}_{2}$ & $\mathrm{CO}$ & $\mathbf{N H}_{3}$ & NOx $_{x}$ & EC & OC & $\mathbf{P M}_{2.5}$ & $\mathbf{P M}_{10}$ & NMVOCs & $\mathrm{SO}_{2}$ & $\mathrm{CO}$ & $\mathrm{NH}_{3}$ & $\mathrm{NO}_{\mathrm{x}}$ & EC & OC & $\mathbf{P M}_{2.5}$ & $\mathbf{P M}_{10}$ \\
\hline \multirow[t]{10}{*}{ EP1 } & Shanghai & 0 & 0 & 0 & 0 & 0 & 0 & 0 & 0 & 0 & 0 & 0 & 0 & 0 & 0 & 0 & 0 & 0 & 0 \\
\hline & Jiangsu & 8 & 0 & 29 & 1 & 1 & 5 & 25 & 44 & 54 & 8 & 0 & 28 & 1 & 1 & 5 & 23 & 41 & 49 \\
\hline & Zhejiang & 30 & 1 & 182 & 6 & 4 & 26 & 287 & 553 & 634 & 11 & 0 & 38 & 1 & 1 & 7 & 31 & 55 & 67 \\
\hline & Anhui & 16 & 0 & 61 & 2 & 2 & 11 & 55 & 97 & 118 & 16 & 0 & 57 & 2 & 2 & 10 & 47 & 83 & 100 \\
\hline & Fujian & 21 & 0 & 119 & 4 & 4 & 19 & 161 & 305 & 364 & 13 & 0 & 45 & 2 & 2 & 8 & 37 & 66 & 79 \\
\hline & Jiangxi & 17 & 0 & 98 & 3 & 3 & 16 & 146 & 263 & 311 & 10 & 0 & 36 & 1 & 1 & 6 & 30 & 53 & 64 \\
\hline & Shandong & 37 & 0 & 136 & 4 & 5 & 24 & 116 & 208 & 254 & 36 & 0 & 128 & 4 & 5 & 22 & 106 & 187 & 226 \\
\hline & Henan & 80 & 0 & 289 & 10 & 11 & 50 & 245 & 434 & 528 & 79 & 0 & 279 & 10 & 10 & 49 & 232 & 408 & 494 \\
\hline & Hubei & 20 & 0 & 79 & 3 & 3 & 14 & 77 & 133 & 163 & 19 & 0 & 69 & 2 & 3 & 12 & 57 & 101 & 122 \\
\hline & Hunan & 2 & 0 & 9 & 0 & 0 & 2 & 10 & 17 & 21 & 2 & 0 & 7 & 0 & 0 & 1 & 6 & 11 & 13 \\
\hline \multirow[t]{10}{*}{ EP2 } & Shanghai & 12 & 0 & 52 & 2 & 2 & 9 & 57 & 98 & 122 & 11 & 0 & 39 & 1 & 1 & 7 & 32 & 57 & $\overline{69}$ \\
\hline & Jiangsu & 221 & 1 & 812 & 27 & 30 & 141 & 710 & 1260 & 1536 & 215 & 1 & 762 & 26 & 28 & 133 & 634 & 1115 & 1349 \\
\hline & Zhejiang & 79 & 1 & 439 & 14 & 13 & 69 & 626 & 1154 & 1357 & 48 & 0 & 171 & 6 & 6 & 30 & 143 & 251 & 304 \\
\hline & Anhui & 1355 & 8 & 4834 & 164 & 179 & 841 & 4067 & 7161 & 8678 & 1347 & 8 & 4778 & 163 & 177 & 832 & 3979 & 6993 & 8464 \\
\hline & Fujian & 48 & 1 & 311 & 10 & 9 & 46 & 499 & 938 & 1111 & 17 & 0 & 59 & 2 & 2 & 10 & 49 & 86 & 105 \\
\hline & Jiangxi & 26 & 1 & 163 & 5 & 4 & 24 & 260 & 488 & 567 & 10 & 0 & 36 & 1 & 1 & 6 & 30 & 52 & 63 \\
\hline & Shandong & 262 & 2 & 950 & 32 & 35 & 166 & 804 & 1425 & 1733 & 259 & 1 & 919 & 31 & 34 & 160 & 766 & 1346 & 1629 \\
\hline & Henan & 833 & 5 & 2983 & 101 & 111 & 519 & 2500 & 4411 & 5351 & 829 & 5 & 2941 & 100 & 109 & 512 & 2449 & 4304 & 5209 \\
\hline & Hubei & 13 & 0 & 54 & 2 & 2 & 9 & 60 & 108 & 129 & 11 & 0 & 39 & 1 & 1 & 7 & 32 & 56 & 68 \\
\hline & Hunan & 14 & 0 & 84 & 3 & 2 & 13 & 128 & 239 & 281 & 7 & 0 & 24 & 1 & 1 & 4 & 20 & 35 & 42 \\
\hline \multirow[t]{10}{*}{ EP3 } & Shanghai & 0 & 0 & 0 & 0 & 0 & 0 & 0 & 0 & 0 & 0 & 0 & 0 & 0 & 0 & 0 & 0 & 0 & $\overline{0}$ \\
\hline & Jiangsu & 7 & 0 & 29 & 1 & 1 & 5 & 29 & 51 & 62 & 7 & 0 & 24 & 1 & 1 & 4 & 20 & 35 & 43 \\
\hline & Zhejiang & 12 & 0 & 64 & 2 & 2 & 10 & 93 & 177 & 205 & 6 & 0 & 21 & 1 & 1 & 4 & 18 & 31 & 38 \\
\hline & Anhui & 38 & 0 & 138 & 5 & 5 & 24 & 117 & 208 & 253 & 37 & 0 & 132 & 5 & 5 & 23 & 110 & 194 & 235 \\
\hline & Fujian & 3 & 0 & 20 & 1 & 1 & 3 & 27 & 47 & 59 & 2 & 0 & 8 & 0 & 0 & 1 & 7 & 12 & 14 \\
\hline & Jiangxi & 30 & 1 & 197 & 6 & 5 & 29 & 321 & 603 & 697 & 10 & 0 & 37 & 1 & 1 & 6 & 30 & 53 & 65 \\
\hline & Shandong & 7 & 0 & 28 & 1 & 1 & 5 & 25 & 45 & 55 & 7 & 0 & 24 & 1 & 1 & 4 & 20 & 35 & 42 \\
\hline & Henan & 15 & 0 & 56 & 2 & 2 & 10 & 47 & 84 & 102 & 15 & 0 & 54 & 2 & 2 & 9 & 45 & 78 & 95 \\
\hline & Hubei & 4 & 0 & 14 & 0 & 1 & 2 & 14 & 26 & 32 & 3 & 0 & 11 & 0 & 0 & 2 & 9 & 16 & 19 \\
\hline & Hunan & 27 & 1 & 200 & 6 & 5 & 28 & 339 & 649 & 748 & 5 & 0 & 16 & 1 & 1 & 3 & 14 & 24 & 29 \\
\hline
\end{tabular}


Table S5. The comparisons of the simulated PM $\mathrm{PM}_{2.5}$ composition $\left(\mu \mathrm{g} \mathrm{m}^{-3}\right)$ in the BASE and OPT cases with the observations $(\mathrm{OBS})$ as well as their corresponding NMB values (\%).

\begin{tabular}{|c|c|c|c|c|c|c|c|c|c|c|c|c|}
\hline \multirow{2}{*}{ Composition } & \multirow{2}{*}{ Time periods } & & \multicolumn{2}{|c|}{ Changsha } & \multicolumn{2}{|c|}{ Hefei } & \multicolumn{2}{|c|}{ Yucheng } & \multicolumn{2}{|c|}{ Qianyanzhou } & \multicolumn{2}{|c|}{ Wuxi } \\
\hline & & & Values & NMB & Values & NMB & Values & NMB & Values & NMB & Values & NMB \\
\hline \multirow{9}{*}{$\mathbf{K}+$} & \multirow{3}{*}{$\begin{array}{l}\text { From } 10 \text { a.m., June } 2 \\
\text { to } 10 \text { a.m., June } 4\end{array}$} & OBS & 0.59 & & 0.92 & & 0.90 & & 0.09 & & 0.62 & \\
\hline & & BASE & 0.15 & -74.64 & 0.18 & -80.53 & 0.35 & -61.12 & 0.22 & 149.12 & 0.12 & -80.73 \\
\hline & & OPT & 0.21 & -64.50 & 0.28 & -69.71 & 0.45 & -50.01 & 0.25 & 183.09 & 0.13 & -79.12 \\
\hline & \multirow{3}{*}{$\begin{array}{l}\text { From } 10 \text { a.m., June } 9 \\
\text { to } 10 \text { a.m., June } 11\end{array}$} & OBS & 1.50 & & 1.16 & & 1.49 & & 0.56 & & 0.56 & \\
\hline & & BASE & 0.49 & -67.34 & 0.55 & -52.49 & 0.78 & -47.67 & 0.41 & -26.80 & 0.38 & -32.23 \\
\hline & & OPT & 0.79 & -47.34 & 0.89 & -23.12 & 0.87 & -41.63 & 0.45 & -19.65 & 0.34 & -39.37 \\
\hline & \multirow{3}{*}{$\begin{array}{l}\text { From } 10 \text { a.m., June } 16 \\
\text { to } 10 \text { a.m., June } 18\end{array}$} & OBS & 0.51 & & 0.91 & & 0.51 & & 0.16 & & 0.25 & \\
\hline & & BASE & 0.34 & -33.36 & 0.54 & -40.52 & 0.71 & 39.78 & 0.29 & 77.65 & 0.07 & -72.05 \\
\hline & & OPT & 0.38 & -25.52 & 0.78 & -14.09 & 0.74 & 45.68 & 0.33 & 102.15 & 0.09 & -64.07 \\
\hline \multirow{9}{*}{$\mathrm{SO}_{4}{ }^{2-}$} & \multirow{3}{*}{$\begin{array}{l}\text { From } 10 \text { a.m., June } 2 \\
\text { to } 10 \text { a.m., June } 4\end{array}$} & OBS & 20.48 & & 10.95 & & 13.11 & & 12.56 & & 16.21 & \\
\hline & & BASE & 11.40 & -44.34 & 5.90 & -46.12 & 8.70 & -33.64 & 1.98 & -84.24 & 9.64 & -40.54 \\
\hline & & OPT & 24.12 & 17.77 & 13.54 & 23.64 & 15.34 & 17.01 & 3.45 & -72.53 & 9.58 & -40.91 \\
\hline & \multirow{3}{*}{$\begin{array}{l}\text { From } 10 \text { a.m., June } 9 \\
\text { to } 10 \text { a.m., June } 11\end{array}$} & OBS & 33.83 & & 18.68 & & 30.74 & & 8.97 & & 13.23 & \\
\hline & & BASE & 20.14 & -40.47 & 14.70 & -21.31 & 15.98 & -48.01 & 2.55 & -71.56 & 8.40 & -36.53 \\
\hline & & OPT & 37.07 & 9.57 & 22.30 & 19.38 & 31.99 & 4.07 & 3.54 & -60.52 & 9.00 & -31.99 \\
\hline & \multirow{3}{*}{$\begin{array}{l}\text { From } 10 \text { a.m., June } 16 \\
\text { to } 10 \text { a.m., June } 18\end{array}$} & OBS & 18.94 & & 27.05 & & 21.86 & & 20.95 & & 12.00 & \\
\hline & & BASE & 13.75 & -27.39 & 16.70 & -38.26 & 8.71 & -60.15 & 4.78 & -77.19 & 6.85 & -42.90 \\
\hline & & OPT & 23.64 & 24.83 & 28.90 & 6.85 & 18.05 & -17.41 & 5.61 & -73.23 & 7.40 & -38.31 \\
\hline \multirow{9}{*}{$\mathrm{NO}_{3}{ }^{-}$} & \multirow{3}{*}{$\begin{array}{l}\text { From } 10 \text { a.m., June } 2 \\
\text { to } 10 \text { a.m., June } 4\end{array}$} & OBS & 9.34 & & 8.48 & & 7.49 & & 1.93 & & 6.35 & \\
\hline & & BASE & 10.97 & 17.49 & 5.39 & -36.44 & 3.61 & -51.81 & 1.03 & -46.56 & 1.78 & -71.96 \\
\hline & & OPT & 14.98 & 60.44 & 9.81 & 15.67 & 6.45 & -13.90 & 2.62 & 35.94 & 1.95 & -69.28 \\
\hline & \multirow{3}{*}{$\begin{array}{l}\text { From } 10 \text { a.m., June } 9 \\
\text { to } 10 \text { a.m., June } 11\end{array}$} & OBS & 11.57 & & 3.20 & & 12.72 & & 0.60 & & 1.82 & \\
\hline & & BASE & 9.70 & -16.18 & 7.90 & 146.66 & 10.80 & -15.13 & 1.85 & 207.90 & 0.98 & -46.05 \\
\hline & & OPT & 12.45 & 7.58 & 8.50 & 165.40 & 14.45 & 13.56 & 2.95 & 390.98 & 0.97 & -46.60 \\
\hline & \multirow{3}{*}{$\begin{array}{l}\text { From } 10 \text { a.m., June } 16 \\
\text { to } 10 \text { a.m., June } 18\end{array}$} & OBS & 4.85 & & 28.33 & & 15.89 & & 2.22 & & 12.27 & \\
\hline & & BASE & 7.40 & 52.45 & 20.00 & -29.39 & 20.38 & 28.23 & 1.88 & -15.36 & 14.95 & 21.82 \\
\hline & & OPT & 8.78 & 80.88 & 29.80 & 5.21 & 21.09 & 32.69 & 2.23 & 0.40 & 15.78 & 28.59 \\
\hline \multirow{9}{*}{$\mathbf{N H}_{4}{ }^{+}$} & \multirow{3}{*}{$\begin{array}{l}\text { From } 10 \text { a.m., June } 2 \\
\text { to } 10 \text { a.m., June } 4\end{array}$} & OBS & 10.26 & & 9.03 & & 8.45 & & 5.46 & & 9.17 & \\
\hline & & BASE & 8.45 & -17.63 & 7.54 & -16.53 & 3.78 & -55.27 & 1.03 & -81.14 & 13.50 & 47.27 \\
\hline & & OPT & 11.54 & 12.50 & 10.92 & 20.89 & 7.30 & -13.61 & 1.75 & -67.96 & 13.90 & 51.64 \\
\hline & \multirow{3}{*}{$\begin{array}{l}\text { From } 10 \text { a.m., June } 9 \\
\text { to } 10 \text { a.m., June } 11\end{array}$} & OBS & 17.68 & & 8.54 & & 15.26 & & 3.71 & & 4.76 & \\
\hline & & BASE & 11.06 & -37.45 & 4.98 & -41.72 & 13.45 & -11.88 & 1.35 & -63.59 & 2.01 & -57.77 \\
\hline & & OPT & 17.37 & -1.76 & 10.39 & 21.60 & 18.74 & 22.78 & 1.75 & -52.80 & 3.32 & -30.25 \\
\hline & & OBS & 8.62 & & 21.87 & & 14.95 & & 10.14 & & 9.74 & \\
\hline & From 10 a.m., June 16 & BASE & 3.84 & -55.47 & 20.06 & -8.29 & 14.20 & -4.99 & 5.97 & -41.11 & 8.70 & -10.65 \\
\hline & & OPT & 10.43 & 20.94 & 28.78 & 31.57 & 17.64 & 18.03 & 6.78 & -33.12 & 8.40 & -13.73 \\
\hline & & OBS & 19.80 & & 19.54 & & 18.95 & & 15.70 & & 29.15 & \\
\hline OC & From 10 a.m., June 9 & BASE & 10.15 & -48.74 & 10.30 & -47.30 & 10.65 & -43.81 & 6.12 & -61.02 & 15.07 & -48.30 \\
\hline & to 10 a.m., June 11 & OPT & 18.50 & -6.57 & 15.50 & -20.70 & 13.50 & -28.77 & 8.12 & -48.28 & 15.95 & -45.28 \\
\hline
\end{tabular}


Table S6. The NMB and R values between observed and simulated surface chemical species over CEC in the BASE and OPT cases.

\begin{tabular}{l|ccccc|ccccc}
\hline \multirow{2}{*}{ Provinces } & \multicolumn{6}{|c|}{ BASE case (NMB \% /R) } & \multicolumn{4}{c}{ OPT case (NMB \% /R) } \\
\cline { 2 - 10 } & $\mathbf{C O}$ & $\mathbf{S O}_{\mathbf{2}}$ & $\mathbf{N O}_{\mathbf{2}}$ & $\mathbf{P M}_{\mathbf{2 . 5}}$ & $\mathbf{P M}_{\mathbf{1 0}}$ & $\mathbf{C O}$ & $\mathbf{S O}_{\mathbf{2}}$ & NO $_{\mathbf{2}}$ & PM$_{\mathbf{2 . 5}}$ & PM$_{\mathbf{1 0}}$ \\
\hline Henan & $-22.34 / 0.75$ & $-29.05 / 0.69$ & $-5.33 / 0.71$ & $-24.73 / 0.74$ & $-20.89 / 0.78$ & $-12.25 / 0.89$ & $-22.23 / 0.79$ & $3.02 / 0.78$ & $-1.28 / 0.90$ & $-2.03 / 0.89$ \\
Anhui & $-20.67 / 0.71$ & $-28.02 / 0.71$ & $-7.56 / 0.69$ & $-19.32 / 0.70$ & $-22.49 / 0.72$ & $5.19 / 0.88$ & $-26.85 / 0.81$ & $-99.97 / 0.79$ & $4.18 / 0.80$ & $6.11 / 0.84$ \\
Hubei & $-29.53 / 0.41$ & $-30.35 / 0.42$ & $-18.55 / 0.33$ & $-32.97 / 0.31$ & $-31.83 / 0.41$ & $-13.61 / 0.62$ & $-22.09 / 0.61$ & $-12.59 / 0.42$ & $-2.23 / 0.53$ & $-3.06 / 0.61$ \\
Hunan & $-11.99 / 0.63$ & $-15.73 / 0.62$ & $-17.01 / 0.66$ & $-10.56 / 0.66$ & $-15.62 / 0.69$ & $9.08 / 0.81$ & $-19.06 / 0.77$ & $-17.13 / 0.76$ & $8.21 / 0.74$ & $6.23 / 0.79$ \\
Jiangxi & $-14.86 / 0.65$ & $-19.54 / 0.59$ & $-17.96 / 0.61$ & $-7.15 / 0.59$ & $-12.63 / 0.61$ & $6.39 / 0.71$ & $-20.05 / 0.68$ & $-19.98 / 0.78$ & $5.22 / 0.56$ & $7.16 / 0.62$ \\
Zhejiang & $-17.03 / 0.79$ & $-17.63 / 0.75$ & $-17.36 / 0.77$ & $-5.23 / 0.81$ & $-11.53 / 0.77$ & $4.22 / 0.86$ & $-19.35 / 0.79$ & $19.08 / 0.86$ & $3.45 / 0.80$ & $3.45 / 0.85$ \\
Fujian & $-29.63 / 0.74$ & $-36.49 / 0.66$ & $-36.86 / 0.69$ & $-39.00 / 0.67$ & $-24.51 / 0.77$ & $-11.06 / 0.68$ & $-36.52 / 0.75$ & $-28.59 / 0.81$ & $-22.00 / 0.64$ & $-16.03 / 0.77$ \\
Shandong & $-26.33 / 0.77$ & $-33.15 / 0.57$ & $-16.39 / 0.67$ & $-28.51 / 0.84$ & $-27.61 / 0.81$ & $-15.04 / 0.80$ & $-33.89 / 0.69$ & $-17.16 / 0.70$ & $-17.89 / 0.82$ & $-12.09 / 0.86$ \\
Jiangsu & $-28.03 / 0.73$ & $-38.22 / 0.48$ & $-27.43 / 0.60$ & $-37.08 / 0.85$ & $-33.49 / 0.83$ & $-13.23 / 0.79$ & $-38.00 / 0.77$ & $-25.51 / 0.66$ & $-29.17 / 0.79$ & $-11.92 / 0.88$ \\
Shanghai & $-24.49 / 0.81$ & $-30.15 / 0.52$ & $-6.07 / 0.63$ & $-38.05 / 0.79$ & $-30.09 / 0.73$ & $-11.01 / 0.87$ & $-26.85 / 0.81$ & $-5.08 / 0.67$ & $-13.63 / 0.81$ & $-9.16 / 0.79$ \\
\hline
\end{tabular}


80 Table S7. The NMB and $R$ values between observed and simulated surface meteorological species over CEC in the OPT case.

\begin{tabular}{|c|c|c|c|c|}
\hline \multirow{2}{*}{ Provinces } & \multicolumn{4}{|c|}{ OPT case (NMB \%/R) } \\
\hline & Temperature & Relative humidity & Wind speed & PBL height \\
\hline Henan & $-0.03 / 0.87$ & $-0.05 / 0.71$ & $0.19 / 0.61$ & $-2.13 / 0.63$ \\
\hline Anhui & $0.06 / 0.72$ & $-0.21 / 0.55$ & $0.08 / 0.41$ & $3.18 / 0.66$ \\
\hline Hubei & $-0.04 / 0.85$ & $0.31 / 0.72$ & $0.48 / 0.49$ & $2.19 / 0.71$ \\
\hline Hunan & $-0.11 / 0.79$ & $-0.24 / 0.67$ & $0.96 / 0.49$ & $3.49 / 0.51$ \\
\hline Jiangxi & $-0.28 / 0.80$ & $-0.12 / 0.64$ & $0.60 / 0.52$ & $2.96 / 0.55$ \\
\hline Zhejiang & $-0.12 / 0.83$ & $-0.12 / 0.72$ & $0.90 / 0.38$ & $-3.05 / 0.48$ \\
\hline Fujian & $-0.33 / 0.84$ & $-0.36 / 0.66$ & $0.75 / 0.52$ & $4.96 / 0.67$ \\
\hline Shandong & $-0.36 / 0.81$ & $-0.42 / 0.63$ & $0.66 / 0.62$ & $3.59 / 0.41$ \\
\hline Jiangsu & $-0.29 / 0.74$ & $-0.41 / 0.64$ & $0.86 / 0.55$ & $4.03 / 0.62$ \\
\hline Shanghai & $-0.39 / 0.69$ & $-0.45 / 0.59$ & $0.94 / 0.71$ & $4.11 / 0.59$ \\
\hline
\end{tabular}

